\title{
La modelación metafórica del movimiento por estudiantes universitarios
}

\author{
Elizabeth H. Arredondo, Jaime I. García-García y Maximina Márquez Torres \\ Universidad de Los Lagos, Dpto. de Ciencias Exactas, Av. Alberto Fuchslocher 1305, Osorno, Chile. \\ (correo-e: elizabeth.hernandez@ulagos.cl; jaime.garcia@ulagos.cl; maximina.marquez@ulagos.cl)
}

Recibido Sep. 3, 2019; Aceptado Oct. 29, 2019; Versión final Ene. 16, 2020, Publicado Jun. 2020

\begin{abstract}
Resumen
Este artículo identifica las metáforas conceptuales que están presentes en los procesos de modelización asociadas al objeto matemático 'vector' cuando un grupo de estudiantes de ingeniería resuelve tareas de velocidad relativa. El interés nace al identificar que este objeto articula magnitudes físicas, como velocidad y fuerza, con propiedades matemáticas, y proporciona un campo de representación que respalda su interpretación. Se utilizó una metodología cualitativa de carácter interpretativo y descriptivo. Se consideraron como unidades de análisis las respuestas dadas por los estudiantes de forma oral, gestual, escrita o representadas en la computadora. Se observó que el manejo correcto de las operaciones básicas del álgebra apoya la resolución de problemas físicos. Pero, la interpretación adecuada de los fenómenos involucrados requiere de una modelación apuntalada en el 'sistema de referencia' y del uso de metáforas que vinculen el fenómeno de movimiento con las propiedades físicas y matemáticas.
\end{abstract}

Palabras clave: modelación; vector; metáfora; movimiento

\section{Metaphorical modeling of movement by university students}

\begin{abstract}
This article identifies the conceptual metaphors present in the modeling processes associated with the mathematical object "vector" when a group of engineering students solve tasks of relative velocity. The interest on this originated after identifying that the object "vector" articulates physical quantities such as velocity and force. This object also has mathematical properties and provides a field for representations that supports their interpretations. The present study was based on a qualitative methodology of interpretative and descriptive nature that used as units of analysis the answers provided by students in the following manners: verbal, in gestures, written, or on a computer. It was observed that the correct management of algebra's basic operations supports the resolution of physical problems. But, the proper interpretation of the phenomena involved requires modeling supported on the "reference system" and requires the use of metaphors that link the phenomenon of movement with physical and mathematical properties.
\end{abstract}

Keywords: modeling; vector; metaphors; movement 


\section{INTRODUCCIÓN}

Para la mayoría de los estudiantes de ingeniería, es común que en los primeros años de su formación encuentren cursos, tanto de Matemáticas como de Física, donde se use al objeto matemático 'vector' y, a pesar de que este objeto es compartido a través de sus representaciones algebraica, gráfica y en su operatividad, inicialmente los universitarios guardan la idea que se trabaja con objetos distintos (Acuña, Hernández y Liern, 2017; Niño, Herrera y Gómez, 2006). Esta confusión puede deberse a fenómenos propios del lenguaje matemático, que hacen uso de representaciones del mismo objeto o relaciones matemáticas, o de la misma representación para denotar distintos objetos (Serrano, 2005).

En el caso del vector, usado en álgebra lineal, se apoya en la teoría de espacios vectoriales; mientras que el empleado en física privilegia las ideas de ser una flecha que tiene: punto de origen, punto final, magnitud, dirección y sentido. Esta es una idea acotada del espacio vectorial, privilegiando su representación en segunda y tercera dimensión del campo de los reales y, que posee la representación gráfica de una flecha (Acuña, et al. 2017). El objetivo de este trabajo es identificar las metáforas conceptuales que están presentes en los procesos de modelización asociadas al vector cuando se resuelven problemas de movimiento relativo en una clase de física universitaria; por lo que podemos plantearnos las siguientes preguntas que guían nuestra investigación: 1) ¿Cuáles son las metáforas conceptuales que se encuentran presentes en la modelización vectorial de problemas de movimiento relativo? 2) ¿Cómo es la relación entre el proceso de modelación y la metáfora conceptual que orienta la solución de los problemas de movimiento relativo?

Como parte de los antecedentes de este trabajo de investigación, es necesario aclarar ciertos puntos que permitan plantear adecuadamente el fenómeno didáctico a explorar. Para ello, se desarrollarán los siguientes aspectos: exploración de las dificultades de la naturaleza de las cantidades físicas (escalares y vectoriales); datos históricos sobre los vectores en matemáticas y en física; investigaciones acerca del uso del vector en física; un acercamiento a la propuesta teórica de la investigación.

Las cantidades que se usan en física son escalares y vectoriales, las que, a su vez, son ubicuas debido a que se encuentran presentes en todo momento en la física. Sin embargo, los libros de pregrado solo ofrecen una breve descripción de éstas, lo que causa una serie de dificultades que dejan reducida la naturaleza de la cantidad al segmento de línea dirigida al paralelogramo de fuerza o a la adición triangular, entre otros. De hecho, aun cuando un vector en física puede ser representado a partir de una magnitud y una dirección, no siempre se cumple que toda representación de una magnitud y dirección sea un vector (Joshi y Kumar, 2004). Actualmente los estudiantes deben hacer uso de los vectores como objetos con ciertas propiedades matemáticas y físicas, cuyo comportamiento será de naturaleza algebraica o geométrica, o se pueden referir a ellos gráficamente en espacios vectoriales. En el caso de este trabajo es interesante conocer cómo se establece esta relación de convivencia en el tratamiento de los vectores para modelar y resolver problemas del movimiento dentro de un entorno algebraico y/o geométrico, haciendo uso de una herramienta tecnológica.

El tratamiento de los vectores en la escuela apunta a un primer problema que se relaciona con las distintas formas como se presentan en los entornos físico y matemático, ya que a pesar de que se trata del mismo contenido matemático, la diferencia en la presentación de éste en cada entorno dista mucho de ser natural porque debe pasar por un trabajo de cambio de registro semiótico (Duval, 2010). Esto implica que se debe establecer, además del significado en cada entorno, otro destinado para vincular los significados entre las distintas representaciones. En el caso de que la presentación del vector como flecha sea la más conveniente para ese momento, pudiera ser prudente advertir que esta presentación no es más que una de las diversas formas en las que puede ser representado un vector, aunque el cambio de registro no deja de ser una tarea que debe ser afrontada más adelante.

Los conceptos teóricos y las representaciones usadas en los libros de física exhiben un fenómeno analizado por Núñez (2011), que consiste en referirse a estos objetos matemáticos y/o físicos de forma estática debido a la naturaleza propia de los mismos, pero es en el proceso de enseñanza que el profesor le imprime un significado de tipo dinámico a dichos conceptos, momento en el cual el estudiante asocia a estas propiedades una representación icónica. Este tipo de fenómenos arroja una profunda incompatibilidad cognitiva entre los estudiantes según palabras de Núñez (2011), debido a que el objeto matemático es presentado teóricamente como estático, pero para fines de la enseñanza y aprendizaje, los profesores le imprimen un carácter dinámico que es preferido por nuestros estudiantes, pues se asocia a fenómenos de movimiento.

Con respecto al uso contextual y temático del problema físico encontramos los trabajos de Nguyen y Meltzer (2003) y Shaffer y McDermott (2005). En particular, estos últimos autores realizaron una investigación acerca de las habilidades de los estudiantes universitarios cuando usan vectores de velocidad y aceleración, en una y dos dimensiones, además de su comprensión del movimiento. En ésta se observa que, al momento de realizar interpretaciones sobre diversos sucesos, se tienden a mezclar los significados físicos y matemáticos que los definen con aquellos ligados a elementos de la vida cotidiana y que tienden a ser poco nítidos. También señalan que los estudiantes tienen deficiencias en la interpretación del problema propuesto fundamentalmente porque ignoran o confunden los conceptos físicos involucrados, no obstante, es necesario incluir el aspecto del 
tratamiento ambiguo de los signos involucrados. En paralelo, este mismo objeto matemático ha sido analizado desde el álgebra lineal, recibiendo una serie de críticas por parte de los alumnos y los estudios debido al excesivo formalismo y a la gran cantidad de definiciones que obstaculizan las ideas matemáticas asociadas (Dorier, Robert, Robinet, y Rogalsiu, 2000; Sierpinska, 2000).

En una dirección distinta tenemos el trabajo de Watson, Spyrou y Tall (2004) que trata de la relación entre la corporeidad y el simbolismo matemático del concepto de vector; en donde distingue el vector en distintos aspectos representativos del mundo: el mundo perceptual y el mundo conceptual de simbolismo matemático con particular referencia al concepto de vector, y el mundo perceptible que tiene una variedad de diferentes significados que dependen del contexto en donde este se produce; por lo tanto, su análisis consiste en su transición del mundo real al modelo científico, y éste a su vez al modelo matemático. Watson, et al. (2004) consideran que el aprendizaje de los vectores está situado en la intersección entre la teoría encarnada relativa a los fenómenos físicos y el llamado proceso-objeto de su teoría, el cual se encapsula en las acciones desarrolladas con los conceptos matemáticos.

En el siguiente apartado se presentan los referentes teóricos que dan sustento a la presente investigación, y en el que es de interés observar las relaciones que desarrolla el estudiante entre la metáfora signo-flecha y sus propiedades matemáticas de magnitud, dirección y sentido. Además, se considera como opción interesante a investigar si este vínculo de la metáfora signo-flecha, como prototipo del vector, persiste cuando los estudiantes se ven obligados a darle sentido en distintos registros de representación.

\section{OTROS ANTECEDENTES}

En la sección anterior se hicieron ciertas consideraciones sobre el llamado signo-flecha como representación gráfica del vector, el cual adopta significados inadecuados debido a una temprana conexión entre éste y la indicación de movimiento, además del uso abusivo del lenguaje y la exagerada axiomatización. Esto se abordará bajo las siguientes tres líneas de pensamiento:

Los sistemas de representación y el signo

Según Pierce (1974), los signos siempre están en relación con otros objetos, de los cuales se establece algo en su ausencia. La matemática es esencialmente trabajada a partir de signos que se refieren a otros objetos de una cierta manera. En el contexto de la Educación Matemática se considerará la postura de Radford (2006), quien señala que toda actividad matemática será tratada a través de los signos.

Por otro lado, estos signos matemáticos, además de esenciales, pasan a ser parte del conocimiento, tal como sucede en la codificación, construcción y comunicación del conocimiento matemático. Steinbring (2006) postula que través de ellos podemos considerar la generalidad de este conocimiento, en el sentido de reconocer en un evento una forma específica para proceder frente a otros del mismo tipo. En nuestro contexto de estudio, encontramos que el significado de vector debe ser producido por el alumno a través de un entorno adecuado, en el que el uso de signos sea para mostrar la idea que el vector induce en el estudiante. A su vez, Acuña (2012) indica que los signos permiten hacer una evocación del proceso de la construcción del conocimiento de manera general, como un producto de la regulación a la que fue sometido. Sin embargo, el conflicto que surge se debe al tratamiento que tiene el vector en este entorno, donde al objeto matemático se le da corporeidad a partir de ideas cómo movimiento, fuerza, fenómenos que, si bien son abstractos, la cercanía empírica del ser humano a estos fenómenos en la vida real organiza estos conceptos, y en particular al vector, como una unidad orgánica que apoya la apropiación del objeto abstracto.

\section{La cognición encarnada (embodiment cognition)}

Esta postura da cuenta de la actividad corpórea, en el sentido de que el cuerpo contribuye con el cerebro para realizar vinculaciones del exterior de manera abstracta. Para investigadores como Font y Acevedo (2003), el pensamiento metafórico será entendido como la interpretación de un campo de experiencias en términos de otro ya conocido, centrando la importancia del pensamiento metafórico en la formación de los conceptos matemático, como mencionan Lakoff y Johnson (1991). Un elemento importante en esta teoría es la idea 'esquema-imagen' de Johnson (1987) y Lakoff y Johnson (2003), que "son aquellos que constituyen un modelo y establecen un ordenamiento en nuestras acciones, percepciones y concepciones" (Lakoff y Johnson, 2003, p.85). En esta investigación se entiende a un esquema-imagen como una descripción condensada de la experiencia perceptual que vincula estas experiencias y situaciones físicas con una estructura especifica conceptual.

Como mencionan Lakoff y Núñez (2010), desde el punto de vista de la cognición encarnada, en el caso de la esquema-imagen se tienen los siguientes elementos: "un objeto o entidad en movimiento (trayector), una fuente (origen o punto de partida), un objetivo (diana o punto final) y un camino trayectoria o recorrido (serie de lugares contiguos desde el origen hasta el punto final)" (p. 39). Tanto Lakoff y Johnson (2003) como Lakoff y Núñez (2010), consideran importante la aclaración de algunos términos: 1) la proyección metafórica es el principal mecanismo cognitivo que permite que las entidades matemáticas abstractas se estructuren a través de experiencias corporales; 2) la metáfora será entendida como la comprensión de un objeto o cosa de un dominio 
en términos de otro dominio ; 3) una expresión metafórica es un caso particular de una metáfora conceptual, más aun, las metáforas conceptuales permiten que las expresiones metafóricas se agrupen, refinando la metáfora original.

El mecanismo básico de la metáfora conceptual es la proyección, en un sentido similar al matemático, donde las metáforas operan proyectando un dominio de 'partida' desde la estructura de otro dominio de 'llegada'; por ende, la proyección metafórica es la estructuración de un dominio conceptual en términos de otro, que se encuentra sujeto al principio formal de invariancia planteado por Lakoff (1990), quien asegura que la congruencia entre ambos dominios conceptuales surge de tal modo que la topología cognitiva se preserva entre ambos dominios. Esto constituye una condición restrictiva sobre las proyecciones metafóricas, en el sentido de limitar el modo en el cual los dominios pueden ser proyectados. Por lo tanto, la metáfora se presenta como la manera específica de crear un puente conceptual entre un dominio de partida y uno de llegada, es decir, como las generadoras de cierto "isomorfismo que permite que se transpongan una serie de características y estructuras" (Font y Acevedo, 2003, p. 406.). Lakoff y Núñez (2010), distinguen dos tipos de metáforas conceptuales en matemáticas: (i) Conectadas a tierra (Grounding). Estas pueden considerarse extramatemáticas, en otras palabras, relacionan un dominio de llegada dentro de las matemáticas, con un dominio de partida fuera de ellas; y (ii) De enlace (Linking). Estas se consideran intramatemáticas, tienen su dominio de partida y de llegada en las mismas matemáticas.

En este trabajo en particular, los dominios de partida y de llegada permitirán articular el fenómeno físico con el objeto matemático a partir de la modelación matemática, la cual debe someterse a un proceso de desencapsulación para dar sentido en el entorno físico del fenómeno estudiado.

\section{El modelado}

Hay conceptos que, en la literatura científica, se tienden a considerar como un símil. Por ello, desarrollaremos nuestra postura de lo que entendemos como modelación matemática, basados en Doerr y English (2003), la cual hace referencia al uso representacional, dentro de un sistema, de expresiones que integran la escritura de símbolos, diagramas o gráficos, las que a su vez relacionan operaciones que pueden ser utilizadas para describir y explicar el comportamiento de algunos fenómenos físicos como el de movimiento. En trabajos recientes, se identifica el modelado con tecnología como un agente que potencializa la coordinación entre distintos registros de representación, privilegiando el uso visual de propiedades, recreando el fenómeno como un fenómeno dinámico (Villa-Ochoa, González-Gómez y Carmona-Mesa, 2018; Martínez-Palmera, Combita-Niño y De-La-HozFranco, 2018). Es aquí donde la metáfora aparece como un vehículo de interpretación, en donde su uso se asemeja a la construcción o empleo de modelos, debido a que se requiere abstraer información del mundo cotidiano para darle una representación y solución en un entorno matemático, que posteriormente debe de ser descrito dentro del contexto del fenómeno analizado.

Rivadulla (2006) menciona que la vinculación posible entre modelos y metáforas consiste en que la construcción de un modelo revela relaciones nuevas a partir de otras. A su vez, Tall, et al. (2003), propone que la física debe ser vista no solo como el socio natural de las matemáticas puras, sino también como una conexión para comprensión de modelado, el cual es más que un enlace directo del mundo físico a un marco matemático; ya que es necesario disponer de un modelo "científico" de una situación física que ignora los aspectos menos importantes y se centra en las cuestiones esenciales que se pueden modelar de forma matemática.

Cabe señalar que el concepto de modelo científico se refiere a un conjunto amplio de idealizaciones y abstracciones del mundo real, que comprenden cantidades bien definidas, susceptibles de medición experimental, y que abarca también las leyes básicas sobre las cantidades. Respecto a la modelación científica, Touma (2009) le asocia dos fases principales en el pensamiento científico: (1) la fase inductiva, que es cuando el estudiante interactúa con un fenómeno real, identifica variables y formula hipótesis que darán cuerpo a un modelo y/o teoría con una serie de leyes (propias, no necesariamente validas); (2) la fase deductiva, en la que el estudiante intenta con este modelo no solo satisfacer una cuestión relacionada con el fenómeno de partida, sino también deducir y predecir los resultados de algún experimento. La Figura 1 describe este tipo de modelado.

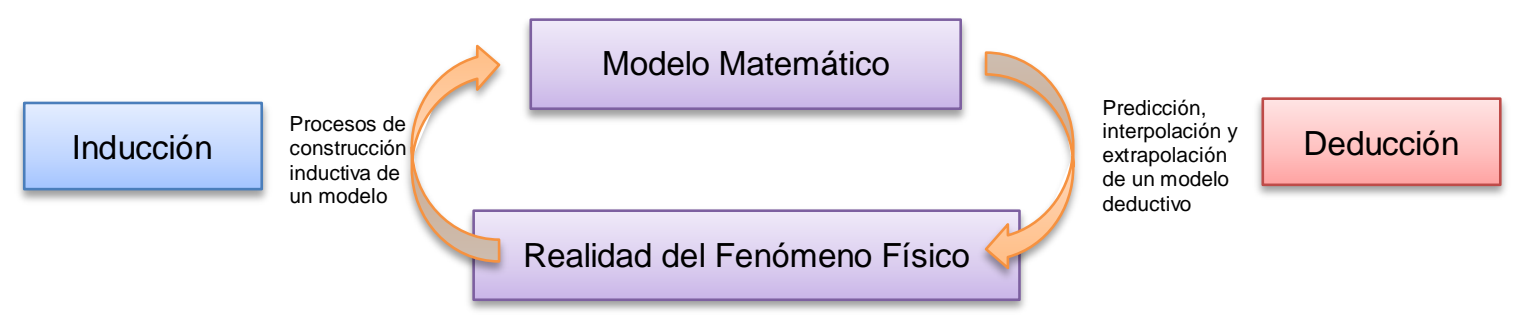

Fig. 1: Modelación matemática de fenómenos científicos, propuesto por Touma.

Por lo tanto, este tipo de modelación facilita el estudio del uso e interpretación de los vectores en problemas de movimiento, aprovechando las representaciones algebraicas y geométricas del objeto vector, pues se enfoca en las estructuras donde se puede hacer uso de patrones y regularidades en los procesos observados. En esta investigación el estudiante presentará el modelado representándolo con apoyo de lápiz y papel, así como con el 
uso de una computadora; esta última le proveerá de un importante apoyo en la fase deductiva, donde además es de interés identificar el tipo de tareas que los estudiantes se proponen para modelar un fenómeno en dos entornos, tanto el matemático como el físico.

\section{METODOLOGÍA}

Este trabajo adoptó una metodología descriptiva-interpretativa que correspondió a un estudio de casos de acuerdo con Cohen, Manion y Morrison (2007), de tipo no participante. Participaron 21 estudiantes, con un promedio de edad de 20 años, quienes cursaban el segundo semestre de la carrera de Licenciatura en Ingeniería en Computación, en una universidad pública de México. Los instrumentos utilizados consistieron en: 1) un cuestionario diagnóstico para identificar sus nociones sobre el vector en física y en matemáticas, 2) ocho actividades semi-guiadas para resolver y modelar problemas de vectores y velocidad relativa, sobre un fenómeno físico de movimiento, donde podían usar el software GeoGebra, y 3) una entrevista por equipos de trabajo donde se exploraban algunos de los problemas trabajados antes (ver Figura 2). Como puede observarse en la figura, los dos primeros momentos de reflexión de la modelización se apoyaron en la propuesta de Touma (2009); y el tercero es el resultado natural de la aprehensión de los dos primeros.

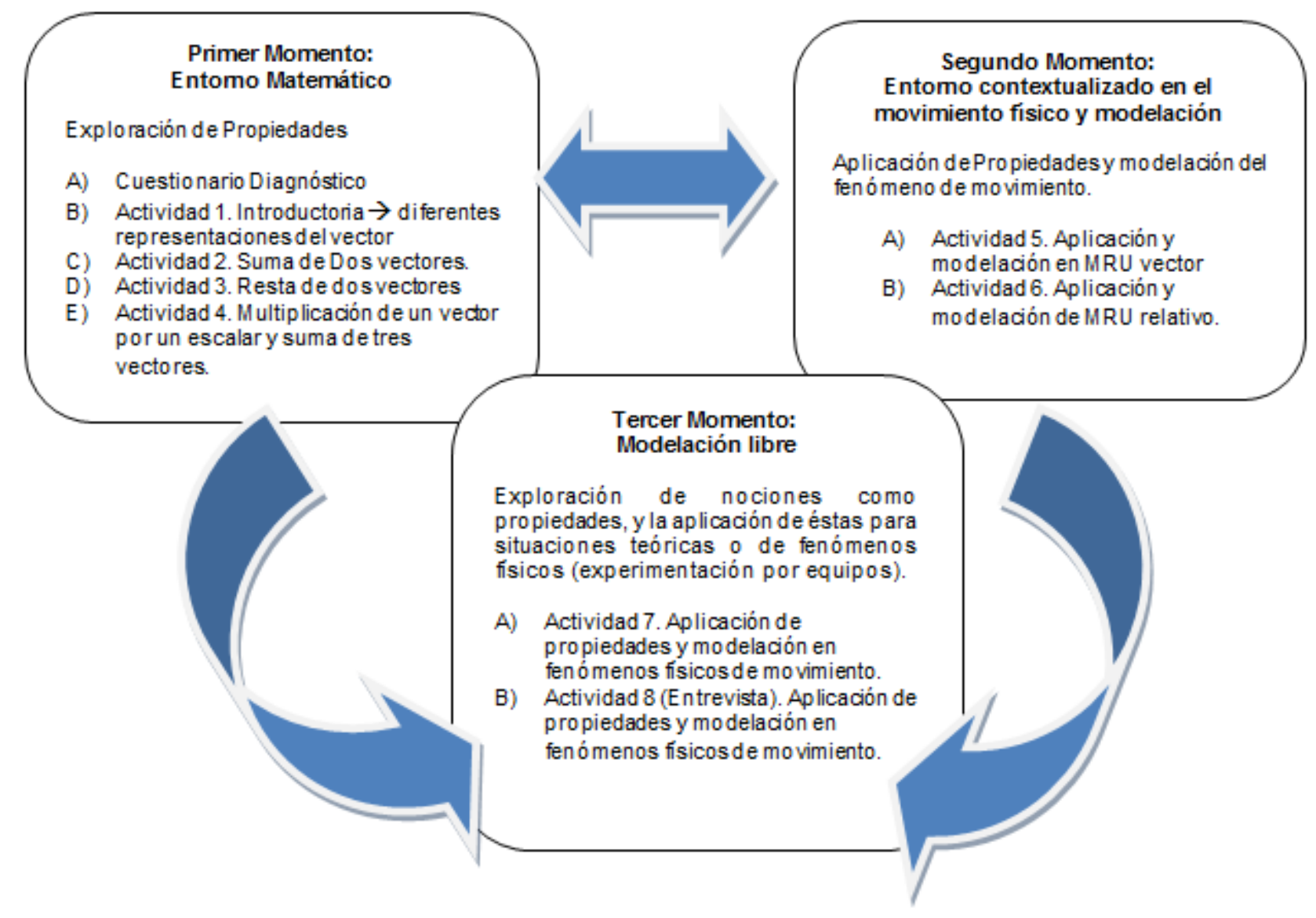

Fig. 2: Diagrama que representa los tres momentos experimentados por los estudiantes durante las actividades propuestas.

Para el planteamiento y la propuesta de las actividades de desplego un análisis curricular de los contenidos que debían desarrollar los estudiantes, esto permitió elegir la población de estudio; una vez planificas las actividades, estas fueron sometidas a validación por triangulación de expertos tanto del área de matemáticas, como de física. Los datos recopilados tuvieron los siguientes formatos: 1) videos de las sesiones de trabajo y entrevistas, 2) registros del cuadernillo de trabajo, por sesión y por equipo, y 3) archivos de trabajo del software GeoGebra. Las unidades de análisis corresponden a un estudio de tipo lingüístico así que, de los instrumentos de recolección, se recuperan párrafos, frases, palabras, gestos, inscripciones y/o dibujos que apoyaran al desarrollo del constructo del vector en el entorno de modelación a partir de las metáforas identificadas, así como las soluciones y modelizaciones desarrolladas por los estudiantes. El tipo de problemas tratados para las actividades y entrevistas se obtuvieron de libros clásicos para la enseñanza de la Física Básica, como el que se muestra en la Figura 3. 
Problema:

Si la velocidad del tren relativa al suelo tiene magnitud $3.0 \mathrm{~m} / \mathrm{s}$ y la velocidad de la mujer relativa al vagón tiene magnitud $1.0 \mathrm{~m} / \mathrm{s}$, mientras que la mujer camina a lo ancho de un vagón de ferrocarril

a) ¿El vector de velocidad relativo al suelo es?

b) Posición de la mujer relativa al marco de referencia del ciclista y al marco del tren

c) El cálculo del desplazamiento de la mujer

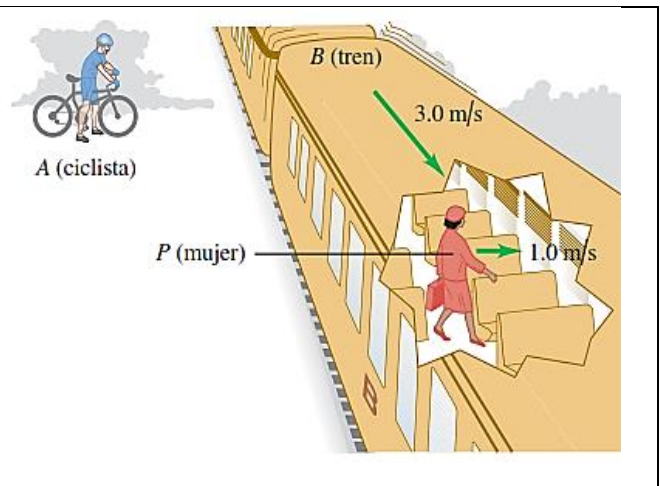

Fig. 3: Tipo de problemas abordados durante la investigación (Zemansky, 2009, p. 91)

\section{RESULTADOS Y DISCUSIÓN}

Los resultados se presentan en tres subsecciones: los entornos matemáticos que apoyan el modelado; el tipo de metáforas que surgen en la explicación del modelado; y ejemplificación de la relación metáfora, modelamiento y tecnología.

\section{Los entornos matemáticos que apoyan el modelado}

Dado el tipo de problemas de velocidad relativa que fueron tratados en este estudio, los entornos de trabajo que se habían considerado eran: gráfico, algebraico y aritmético. Sin embargo, los estudiantes sentían más inclinación por apoyar sus modelamientos en dos de ellos: el gráfico (16) y el algebraico (5). No se puede reportar que tengan un medio de representación favorito, teniendo similar predisposición a usar lápiz y papel, o computadora. En la Tabla 1 se presentan los referentes físicos en que se apoyan los estudiantes para la resolución del problema.

Tabla 1. Tipos de referentes físicos en los que los estudiantes apoyan su reflexión

\begin{tabular}{|l|l|l|l|l|l|l|}
\hline \multicolumn{2}{|l}{ Magnitud } & \multicolumn{2}{l|}{ Dirección } & \multicolumn{2}{l|}{ Sentido } \\
\hline (Vx, Vy $)$ & \multicolumn{1}{|l|}{$|m|$ o $m>0$} & 0 & AD & N, S, E, O & Cuadrante & $+/-$ \\
\hline E2, E10, & E1, E3, E4, E5, E6, & E1, E3, E4, E5, E6, & E2, E10, & E1, E2, E4, E5, E6, & E3, E7, & E8, \\
E11, E17 & E7, E8, E9, E10, E12, & E7, E8, E9, E11, & E17 & E10, E11, E12, E13, & E18 & E18 \\
& E13, E14, E15, E16, & E12, E13, E14, E15, & & E14, E15, E17, E19, & & \\
& E18, E19, E20, E21 & E18, E19, E20, E21 & & E20, E21 & & \\
\hline Total: 4 & Total:18 & Total:17 & Total:3 & Total:15 & Total:3 & Total:2 \\
\hline
\end{tabular}

En la tabla anterior se pueden observar las diferentes representaciones que usaron los estudiantes para modelarlo, donde algunos hacen uso más de una representación para apoyar su solución (como el Estudiante 2 que se apoyó en tres de ellos). Sin embargo, hubo predilección en apoyarse en la idea de magnitud como distancia positiva, de dirección como un ángulo, y de sentido en función a los puntos cardinales. También se desprende que la mayoría de ellos usaron dos formas para representar la magnitud de un vector: usando la computadora, obtuvieron la magnitud en términos de sus componentes rectangulares, las que pueden ser transformadas en un número positivo más sugerente para ellos. De igual forma, se observa que no tienen conflictos con la dirección, asociada con la inclinación del vector medida en grados. Sin embargo, hay conflictos con el sentido del vector, dado que la gran mayoría lo confunde con la dirección. Prefieren usar como referencias los puntos cardinales o bien suponen propiedades que serían correctas si se situaran en el primer cuadrante.

\section{El tipo de metáforas que surgen en la explicación del modelado}

En este apartado se expresarán los resultados obtenidos en función al tipo de metáfora predominante dentro del modelado de este tipo de problemas. En la Tabla 2 se presenta una condensación del mapeo de la información entre el dominio de partida y de llegada de las respuestas de las diferentes actividades desarrolladas por los estudiantes, las cuales se pudieron identificar gracias a su registros escritos, orales, gestuales y de modelación.

En la tabla se observa como el dominio de partida que usaron comienza con la descripción de un fenómeno físico, el cual cobra sentido dentro de las propiedades matemáticas que le permitían modelar y resolver correctamente o no el problema. El tipo de metáforas que se presentan en la modelación se pueden agrupan por rama del conocimiento: (1) Desde el punto de vista del fenómeno físico encontramos el uso de Metáforas de tipo Grounding, donde su dominio de partida está fuera de las matemáticas y se basa en la idea de movimiento en el que se usa espacio hibrido (al mezclar objetos matemáticos con fenómenos físicos), mientras que el dominio de llegada está dentro de las matemáticas y por ello se hace uso de un espacio homogéneo; (2) Desde la matemática, que tiene un carácter más bien abstracto, encontramos una Metáfora de tipo Linking, donde tanto su dominio de partida como su dominio de llegada están formados por conceptos o propiedades matemáticas. 
Tabla 2. Mapeo entre los dominios durante las actividades

\begin{tabular}{|c|c|c|}
\hline Actividades & $\begin{array}{l}\text { Método más usado (Algebraico, } \\
\text { Geométrico), Dominio de Partida }\end{array}$ & Elementos que componen el Dominio de Llegada \\
\hline \multirow{2}{*}{$\begin{array}{l}\text { Previa (2D) } \\
\text { Computacional (2D) } \\
\text { Posterior (2D) }\end{array}$} & $\begin{array}{l}\text { Fuerza, dirección, movimiento, velocidad, } \\
\text { tiempo }\end{array}$ & $\begin{array}{l}\text { Magnitud, dirección, plano cartesiano, origen, } \\
\text { método del polígono y teorema de Pitágoras. }\end{array}$ \\
\hline & $\begin{array}{l}\text { Desplazamiento, punto partida, punto de } \\
\text { llegada, movimiento, velocidad, tiempo, } \\
\text { sistema de referencia, v=d/t, fuerza, } \\
\text { imágenes, sistema de referencia, tiempo. }\end{array}$ & $\begin{array}{l}\text { Magnitud, dirección }\left(^{\circ}\right) \text {, sentido, plano cartesiano, } \\
\text { origen, método por componentes y teorema de } \\
\text { Pitágoras, } v=d / t \text {. }\end{array}$ \\
\hline
\end{tabular}

\section{Ejemplificación de la relación metáfora, modelamiento y tecnología.}

En este punto se exhibirá una serie de imágenes donde los participantes, Pedro $(P)$ y Carlos $(C)$, presentan un tratamiento semiótico del vector. Éste inicia con un tratamiento gestual que es un apoyo para el planteamiento y comprensión de un problema del movimiento relativo, con el fin de mostrar el tratamiento semiótico del signo vector en diferentes momentos de la modelización. Los diálogos presentados son el discurso entablado por los estudiantes, el cual es poco profundo, pero a partir de análisis de ellos podemos identificar el papel que juega la metáfora para guiar la modelación y solución del problema, como se muestra en la Figura 4.

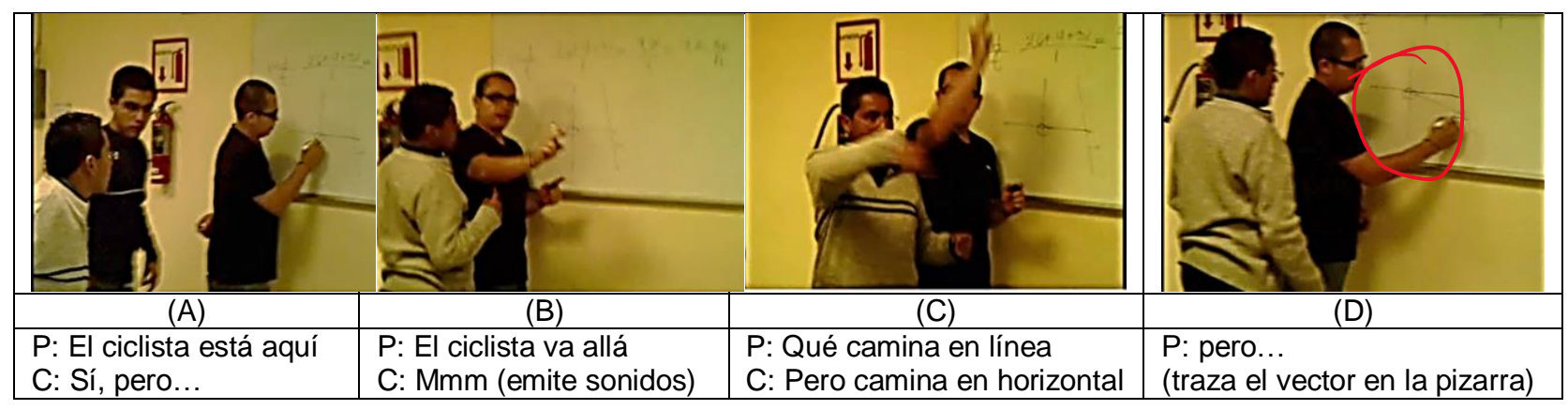

Fig. 4: Tratamiento semiótico del signo vector para el planteamiento del problema

Enseguida se muestra un análisis de los diálogos y escenas presentados en la Figura 4, las cuales son una interpretación de los autores, en donde se presentan elementos del uso de las metáforas y el modelado de los estudiantes en una situación problema. La primera dificultad que surge se refiere a entender e interpretar el movimiento relativo, así como qué elemento deberá ser considerado como punto de referencia para plantear el diagrama vectorial. En la imagen (A), Pedro dibuja un plano cartesiano para indicar el punto de referencia de la observación desde el punto de vista del ciclista. A continuación, imágenes (B) y (C), Pedro y Carlos usan sus manos para indicar donde se coloca la trayectoria del ciclista y la mujer del problema, mientras en la imagen (D) se pueden ver como estos indicadores en los que se han apoyado dan cuerpo a la imagen de la flecha $(\rightarrow)$, dibujada por Pedro en el pizarrón, como un ícono que representa la trayectoria de la mujer que está dentro del vagón. En este primer momento tenemos que la metáfora construida por Pedro se apoya en un signo que indica la dirección. A partir de lo anterior, se presenta la metáfora en la que se apoya la reflexión de Pedro, la cual puede describirse a partir de la idea de la imagen-esquema 'el camino', donde el gesto (movimiento horizontal) representa el caminar en línea horizontal, cuyo significado en el mundo físico hace referencia a la trayectoria que sigue una mujer dentro de un vagón en movimiento, conformando el dominio de partida. Mientras que el dominio de llegada, en el mundo matemático, representa estos movimientos como un vector situado en un sistema de referencia. El primer acercamiento que realizan para plantear el problema no es suficiente para elaborar el diagrama vectorial completo, pues consideran al ciclista en movimiento (el observador bajo el que se sitúa el sistema de referencia).

Esto provoca que Pedro y Carlos reinicien el análisis, apoyados en un tratamiento gestual que permite reorientar el problema. Esto se refleja en la Figura 5, donde también participa Francisco ( $F$ ), en la que se manifiesta la necesidad de Carlos de ubicar un sistema de referencia, para lo cual recurre al uso de la computadora que les permita organizar la modelización del problema y darle sentido a la solución. Es así como el entorno computacional, que apoya la orientación espacial, permite entender a Carlos lo propuesto por sus compañeros, permitiendo organizar los referentes para modelar y solucionar el problema, así como el arribo al entorno matemático de las propiedades de un vector y la interpretación dentro de la física. 


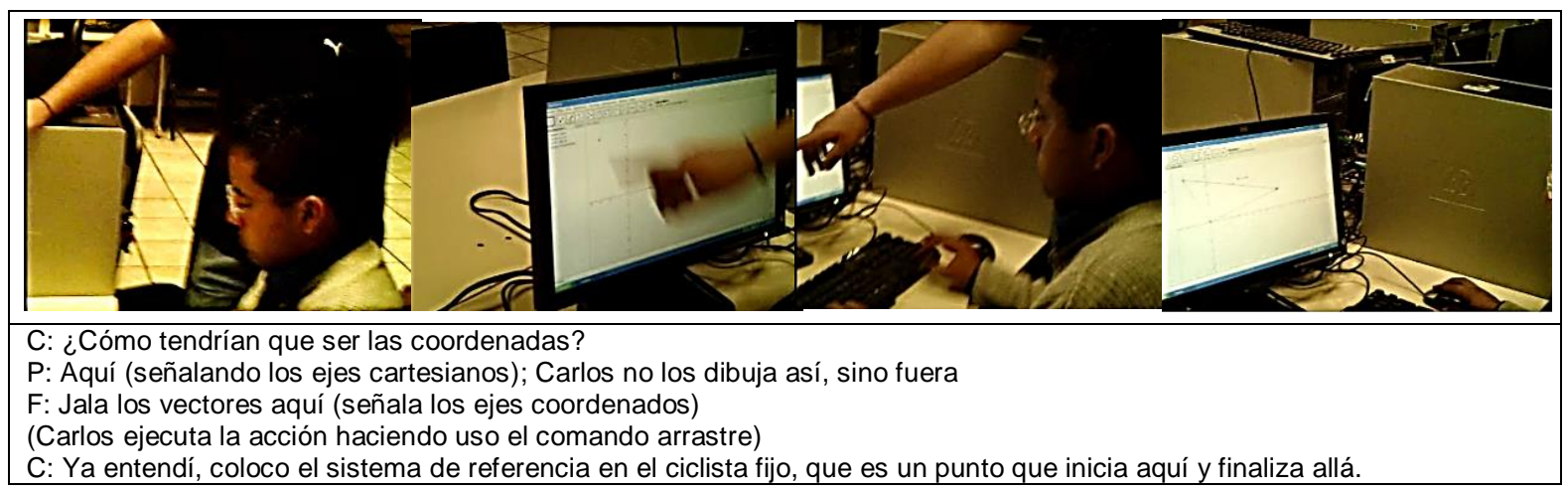

Fig. 5: Reflexión del sistema de referencia apoyándose en la computadora

\section{DISCUSION FINAL}

Los resultados mostrados a lo largo de este artículo presentan la presencia de metáforas (Lakoff y Núñez, 2010; Núñez, 2011) en la solución de las actividades propuestas. La metáfora que está continuamente presente entre los estudiantes para resolver los problemas que requieren representaciones vectoriales corresponden a una de tipo dinámica, a la que denominamos Metáfora del Camino, la que se observa a través de expresiones que hacen referencia al desplazamiento entre el punto inicial y final, donde inicia un vector y donde termina, o como el movimiento en línea recta. De manera general consideramos que esta metáfora es un fundamento en el tratamiento de los vectores como representaciones signo-flecha. Ésta es completada con el uso de otras expresiones (lingüísticas, signos, gestos, entre otros) que se exteriorizan en distintos momentos de la investigación, y su presencia es provocada por las necesidades de dar solución al problema y emergen en la modelación para darle un sentido a esta que, les permita transitar entre los objetos matemáticos a los fenómenos físicos o de los fenómenos físicos a los objetos matemáticos. La Tabla 3 explica la manera en la que se percibe el camino como la interacción de un objeto que se desplaza, de un punto de partida u origen, recorriendo una línea que tiene una direccionalidad y una meta. Hasta aquí se ha desarrollado una síntesis del tipo de metáfora presente en las situaciones experimentadas por los estudiantes.

Tabla 3. Proyecciones entre el esquema de camino y el objeto matemático vector

\begin{tabular}{|l|l|}
\hline Dominio de Partida - Esquema de Camino & Dominio de llegada - Vector \\
\hline Camino (desplazamiento, movimiento) & Magnitud \\
\hline Estar sobre el camino & Dirección \\
\hline Origen del camino & Origen del vector o $(0,0)$ \\
\hline Final del camino & Punto final del vector \\
\hline Recorrido del camino & Sentido \\
\hline Fuera del camino & Elementos que no pertenecen al vector \\
\hline
\end{tabular}

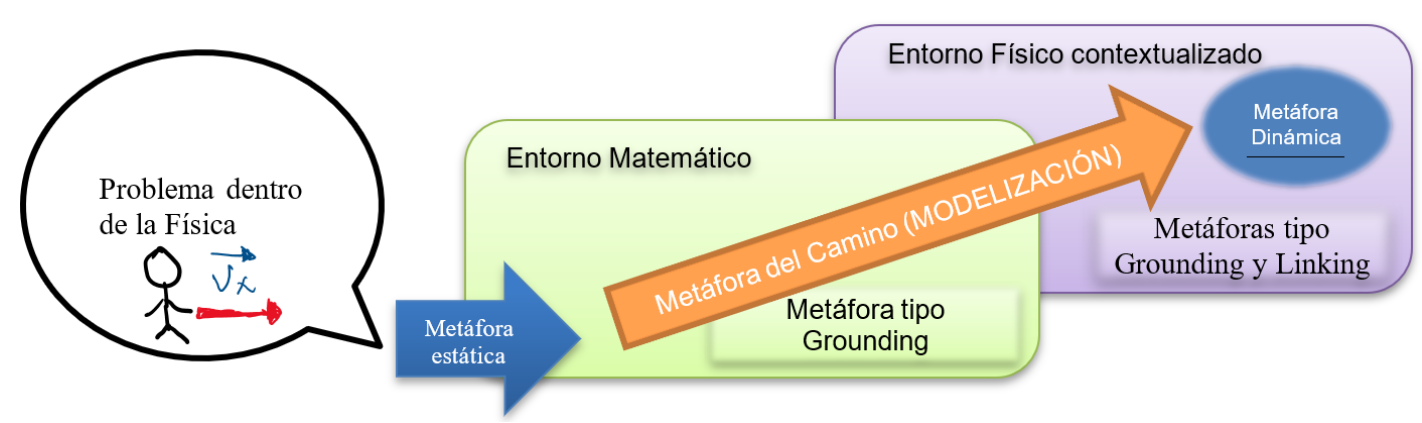

Fig. 6: Esquema de la modelización y la presencia de la metáfora durante la solución de un problema físico

La Figura 6 representa las relaciones presentes en la modelización. Este esquema ejemplifica cómo el problema físico es introducido al entorno matemático a partir de una metáfora estática, en el caso del vector en física, el que se refiere a un vector como un segmento de recta dirigido que tiene un punto de inicio y fin, y el cual cobra sentido en un entorno matemático para ser modelizado (Touma, 2009). A lo largo de este proceso, esta metáfora se torna persistente, apoyada por otras expresiones lingüísticas que daban sentido al problema físico dentro de un contexto. Por ello identificamos que la metáfora se dinamiza en este trayecto de la solución. Finalmente, algo que pudimos observar es que el sistema de referencia es un elemento importante para la solución de este tipo de tareas, ya que una mala ubicación espacial es un elemento que puede obstaculizar la modelización. Por tanto, se torna relevante el papel de la computadora como instrumento de organización espacial de la información y de validador de las soluciones para algunos estudiantes. 


\section{CONCLUSIONES}

De acuerdo con el trabajo presentado y los resultados obtenidos, se pueden plantear las siguientes conclusiones principales:

1. Se identificó una triada de referentes físicos (dirección, magnitud, sentido) sobre los que se apoyan los estudiantes bajo sus diferentes representaciones y significados asociados a los mismos para la solución de problemas. Esta relación tríadica, bajo sus diferentes representaciones, evoca a una metáfora a la que denominamos Metáfora del Camino, que se sustenta en la idea de un punto de inicio, un recorrido, y un punto de fin.

2. La modelación y la metáfora conceptual no son lo mismo, pero tienen una relación que se desdibuja en momentos. A partir de este trabajo identificamos que la metáfora sirve de andamiaje para el proceso de modelización matemática y física; también observamos que, a pesar de tener una misma metáfora, el éxito de la tarea solo se logra con el establecimiento de un sistema de referencia que guie la modelización matemática del fenómeno físico, aquí es donde un factor clave es una correcta interpretación del fenómeno físico.

3. El papel de la computadora en el modelado con tecnología fue imprescindible, pues en su efecto dinámico permite no solo la coordinación el cambio entre registros de representación (Villa-Ochoa, González-Gómez y Carmona-Mesa, 2018; Martínez-Palmera, Combita-Niño y De-La-Hoz-Franco, 2018), sino que además apoya recreando el fenómeno de exploración; este efecto dinámico permite a los estudiantes corregir, en tiempo real, sus apreciaciones sobre la modelación del problema.

\section{REFERENCIAS}

Acuña, C., Hernández, E., y Liern, V., Metáforas conceptuales de las relaciones lineales que manejan los estudiantes de economía, Unión: revista iberoamericana de educación matemática, 50, 29-40 (2017).

Acuña, C., La visualizacion como forma de ver en matematicas: un acercamiento a la investigacion, Gedisa, España (2012).

Black, M., More about metaphor, Metaphor and Thought, Second Edition, https://doi:10.1017/CBO9781139173865.013, Cambridge University Press, Cambridge (1993).

Cohen, L., Manion, L., y Morrison, K., Research methods in education, 6ª Ed., Routledge/Taylor \& Francis, New York, USA (2007).

Doerr, H. M. y English, L. D., A modeling perspective on students' mathematical reasoning about data, https://doi.org/10.2307/30034902, Journal for Research in Mathematics Education 34(2), 110-136 (2003).

Dorier, J. L., Robert, A., Robinet, J., y Rogalsiu, M., The Obstacle of Formalism in Linear Algebra, On the Teaching of Linear Algebra, https://doi.org/10.1007/0-306-47224-4, 85-124, Springer, Luxemburgo (2000).

Duval, R., Sémiosis, pensée humaine et activité mathématique, Amazônia: Revista de Educação em Ciências e Matemáticas, 6(1), 126-143, (2010).

Font, V. y Acevedo, J. I., Fenómenos relacionados con el uso de metáforas en el discurso del profesor. El caso de las gráficas de funciones. Enseñanza de las Ciencias, 21(3), 405-418 (2003).

Johnson, M., The body in the mind: The bodily basis of meaning, imagination, and reason, University of Chicago Press, Chicago, USA (1987).

Joshi, A., y Kumar, A., Scalars vectors in physics. https://doi.org/10.1007/BF02834973, Resonance, 9(11), 56-67 (2004).

Lakoff, R. T., Talking Power: The Politics of Language in Our Lives, Basic Books, New York, USA (1990).

Lakoff, G. y Johnson, M., El cuerpo en la mente. Fundamentos corporales del significado, la imaginación y la razón, Edit. Debate, Madrid, España (1991).

Lakoff, G., y Johnson, M., Metaphors we live by, University of Chicago Press, Chicago, USA (2003).

Lakoff, G., y Núñez, R., Where Mathematics Comes From: How the Embodied Mind Brings Mathematics into Being, Basics Books, New York, USA (2010).

Martínez-Palmera, O., Combita-Niño, H. y De-La-Hoz-Franco, E., Mediación de los Objetos Virtuales de Aprendizaje en el Desarrollo de Competencias Matemáticas en Estudiantes de Ingeniería, http://dx.doi.org/10.4067/S071850062018000600063, Formación universitaria,11(6), 63-74 (2018).

Niño, J. V., Herrera, W. y Gómez, S., Acerca de la enseñanza de la física en las carreras de ingeniería, Revista Colombiana de la Física, 38(4), 1919-1422 (2006).

Nguyen, N., y Meltzer, D. Initial understanding of vector concepts among students in introductory physics courses. https://doi.org/10.1119/1.1571831, American Journal of Physics, 71, 630-638 (2003). 
